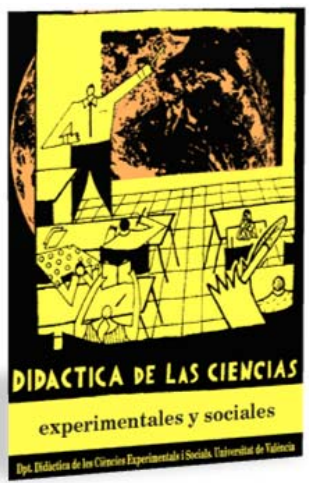

\title{
¿Te gusta conducir? Una mirada crítica desde la publicidad de coches y su retórica medioambiental
}

\author{
Do you like driving? A critical view on car \\ advertising and its environmental rhetoric
}

DOI: $10.7203 / D C E S .34 .11008$

\author{
Miguel García Martín \\ Departamento de Geografía Humana. Universidad de Sevilla \\ mgmartin@us.es \\ ORCID iD: http://orcid.org/0000-0002-3318-4884
}

\begin{abstract}
RESUMEN: El desarrollo sostenible —así como algunos otros relatos en la órbita de la conservación medioambiental - suele utilizarse retóricamente como recurso publicitario para mejorar una imagen de marca. En esa línea, se presenta aquí una propuesta didáctica basada en el análisis crítico del discurso de una muestra de anuncios de televisión. Se presta especial atención a la industria del automóvil, por tratarse de una actividad con enormes repercusiones medioambientales, así como por su impacto mediático y en la cultura popular. Se pone así de manifiesto que en torno al coche siguen gravitando discursos persuasivos más emocionales que racionales, como forma de soslayar el verdadero debate en torno a los desafíos sociales y ambientales actuales.
\end{abstract}

Palabras Clave: Alfabetización mediática, educación ambiental, desarrollo sostenible, automóvil, publicidad.

AвSTRACT: Sustainable development, as well as other narratives within the framework of environmental conservation, is often used as an advertising resource to improve a brand image. In this paper, a teaching proposal based on a critical analysis of the discourse of a set of television commercials is presented. Specific attention is given to the car industry, due to its huge environmental implications and its impact in mass media and popular culture. This study highlights that persuasive discourses around automobile still persist, which are more emotional than rational, as a way to elude the true debates about today's social and environmental challenges.

KEY-WoRDS: Media literacy, environmental education, sustainable development, motor vehicles, advertising.

Fecha de recepción: noviembre de 2017

Fecha de aceptación: abril de 2018 


\section{INTRODUCCIÓN Y MARCO CONCEPTUAL}

Un campo repleto de calabazas entre pinos y cipreses, una fresca mata de tomates aún mojada por el rocío de la mañana, un chef de cocina que huele y palpa las hortalizas, mientras asegura, voz en off, que selecciona "sobre el terreno las mejores verduras, cultivadas de forma sostenible, garantizando así todo su sabor”. El anuncio corresponde a cremas Knorr, marca que comercializa Unilever, una de las mayores compañías agroalimentarias del mundo — 5.600 millones de euros de ingresos netos en 2016 y 168.000 empleados en todo el mundo (Unilever, 2016)—. Definitivamente, la sostenibilidad está hasta en la sopa. No debe sorprender que existan alimentos sostenibles, al margen de lo que eso signifique. Lo paradójico es que lo anuncie dicha multinacional, sin que quede muy claro cómo se puede cultivar de forma sostenible.

La sostenibilidad, y toda la retórica ambiental asociada a este concepto, ha acabado por instalarse casi en cualquier discurso publicitario. Precisamente por la dificultad para delimitar conceptualmente el sentido y el alcance de lo sostenible, cualquiera puede arrogarse el término sin más problemas. Ese es precisamente el marco en el que se inserta este trabajo. Se presenta aquí un análisis crítico como estrategia didáctica cuyo objetivo es desarrollar una lectura comprometida del discurso ambiental en la publicidad por parte del alumnado. Eso sí, se presta especial atención al sector automovilístico, por tratarse de una actividad que involucra a otras muchas (energía, infraestructuras), así como por su alto impacto en la cultura material y su omnipresencia en el paisaje urbano. Para ello, se recurre a una selección de anuncios y campañas publicitarias en las que aparece, explícitamente o mediante lenguajes retóricos y figurados, un pretendido mensaje de protección del medio ambiente que no se corresponde con una realidad subyacente escondida tras esa amable imagen proyectada.

Antes que nada, será necesario contextualizar algunos conceptos capitales. Los paradigmas y las corrientes conservacionistas, tal y como las conocemos hoy, surgen hacia el último tercio del siglo XX. Especialmente desde la publicación de Los límites del crecimiento (Meadows, Meadows, Randers y Behrens, 1972), se ponen de manifiesto las limitaciones del modelo económico capitalista imperante: el planeta es finito y un desarrollo económico basado en la explotación perpetua de sus recursos no se sostiene por mucho tiempo. Desde entonces, numerosos indicadores, cifras y hechos vienen alertando de esta circunstancia: en los últimos 50 años el ser humano ha transformado los ecosistemas con mayor intensidad que en toda la época humana anterior (Millennium Ecosystem Assessment, 2005); las emisiones antropogénicas de gases de efecto invernadero (GEI) han aumentado un 45 \% en el periodo 1970-2010 (IPCC, 2015); la extracción de agua dulce a nivel mundial ha aumentado a razón de un $1 \%$ al año desde finales de la década de 1980, a pesar de que casi 770 millones de personas no tienen acceso a fuentes mejoradas de agua (WWAP, 2014); etc. En el plano social la cuestión no ha mejorado, por cuanto que la desigualdad económica entre ricos y pobres sigue aumentando: desde 2015, un $1 \%$ de la población mundial posee más riqueza que el 99 \% restante. Es la mayor brecha económica de los últimos 30 años (Keeley, 2015; Hardoon, 2017).

Es en este contexto de "crisis ambiental” cuando surge el concepto del desarrollo sostenible. El conocido como informe Brundtland (Informe de la Comisión Mundial sobre el Medio Ambiente y el Desarrollo) lo propone por primera vez y lo define como "aquel que satisface las necesidades del presente sin comprometer la capacidad de las generaciones futuras de satisfacer sus propias necesidades” (Naciones Unidas, 1987, p. 23). Este nuevo enfoque concilia aparentemente el crecimiento económico basado en la producción y explotación de recursos naturales finitos con la conservación de estos mismos recursos. Sin embargo, prácticamente desde su aparición, surge toda una corriente crítica tanto con la formulación estricta del concepto de desarrollo sostenible (Daly, 1990; Constanza y Patten, 1995) como con el alcance tácito de su significado (López y Méndez, 1996). 
La ambigüedad terminológica del desarrollo sostenible ha favorecido su buena acogida en las esferas políticas y económicas, y el escepticismo con el que lo ha tratado la comunidad científica, precisamente por haber surgido en el seno de grandes instituciones internacionales como un proyecto político y no como un objeto de ciencia (Clément, 2005). El uso retórico del término en el discurso mediático, hasta abusar del mismo, lo ha vaciado en parte de contenido, convirtiéndose en un concepto maleta, útil para transportar ideas y representaciones diversas e incluso contradictorias (Aragonés, Raposo y Izurieta, 2001; Clément, 2005).

Esta es precisamente la estrategia que han adoptado los actores económicos y productivos: lejos de cuestionar públicamente los argumentos conservacionistas, las grandes empresas y grupos transnacionales - responsables directos de las transformaciones del medio y de la explotación de los recursos - han aprovechado la debilidad terminológica implícita en el concepto de "lo sostenible” para abrazar, en apariencia, el discurso ambientalista. Esta práctica, conocida como greenwashing, consiste en proyectar hacia la opinión pública (y los consumidores) una imagen ambientalmente responsable y comprometida de una empresa, actividad o producto, por medio de la publicidad (Vos, 2009; Hallama, Montlló, Rofas y Ciutat, 2011). Todo ello, independientemente del verdadero impacto real que dichas empresas puedan generar en el medio natural. La adopción de este "lavado verde" de imagen es especialmente significativo en aquellos sectores económicos de mayor impacto y consumo de recursos (energía y minería, automoción, industria química, etc.), en bienes de consumo de ámbito doméstico susceptibles de suponer un riesgo para la salud (productos para bebés, productos de limpieza y cosméticos) y también en las grandes corporaciones transnacionales preocupadas tanto o más de su imagen de marca que de sus productos (Klein, 2000).

Estudios como los publicados por TerraChoice Group Inc. (2009, 2010) muestran la tendencia progresiva a incorporar un reclamo ambiental en la publicidad de productos y servicios: en el periodo 1987-2008, el número de anuncios con un sesgo verde se multiplicó por diez en Estados Unidos y Canadá, concentrándose ese incremento en los últimos años del periodo. En el ámbito español, numerosos estudios también se han hecho eco de la progresiva penetración del discurso ambiental en los sectores con una fuerte inversión publicitaria, como es el caso de la energía y la automoción, los electrodomésticos, los servicios turísticos y de transporte, etc. (Heras Hernández, 2012). Por un lado, se pone de manifiesto que lo medioambiental vende (Ruiz y Conde, 2002; Andrés y González, 2010): el medio ambiente supone un valor añadido en el producto o servicio, en tanto que ventaja comparativa, más que como valor intrínseco. Para ello, se recurre al relato emocional más que a la información objetiva, en parte aprovechando la ambigüedad conceptual antes mencionada (Álvarez y Reyes, 2011). En el sector del automóvil esta penetración del discurso ambiental ha sido especialmente significativa desde 2008, motivada, entre otras causas, por el cambio en la normativa fiscal que beneficiaba a los coches con menores emisiones de gases de efecto invernadero (GEI por sus siglas) (López, Andrés y González, 2008; Camps, 2010; Pedrós y Martínez, 2010).

Esto conduce a una situación paradójica: se asiste a un significativo aumento de los contenidos y relatos ambientales en las últimas décadas, que contrasta con la incitación implícita a un comportamiento poco respetuoso con el medio ambiente (Pedrós, 2005). Al fin y al cabo, el acto de comprar - como función litúrgica de la posmoderna sociedad de consumo - es contrario a la actitud conservacionista. La solución aparente para consumir en sintonía con el medio ambiente es adquirir productos o servicios que apelen a esos valores ambientales. Toda una paradoja que, sin embargo, constituye el fundamento mismo de la retórica publicitaria ambiental y sostenible.

Esta contradicción no se ha pasado por alto en el ámbito educativo, que la aprovecha como vehículo para educar en y a través de los medios de comunicación y como una oportunidad para favorecer el pensamiento crítico. Precisamente, es la dimensión crítica basada en el enjuiciamiento y la detección de ideologías y valores la que más peso ha adquirido en las competencias de alfabetización mediática (López y Aguaded, 2015; González, Gonzálvez y Ramírez, 2015). En este 
sentido, algunas intervenciones educativas proponen como tratamiento crítico-analítico el uso de publicidad replicante o contrapublicidad (Pacheco, 2009; Prodi, 2016), por la cual los alumnos deben elaborar mensajes críticos basados en estos mismos sistemas de signos y códigos que emplea la industria publicitaria. En esta misma línea, aunque enfocados al más amplio ámbito del activismo civil, se orienta la iniciativa de los Premios Sombra de Ecologistas en Acción, en la que se denuncian con sarcasmo las peores prácticas publicitarias desde el punto de vista social, ético y ambiental.

Recapitulando, nos encontramos ante un estado de "crisis ambiental”, caracterizado por la insostenibilidad del actual sistema económico capitalista de producción y consumo (utilización de recursos por encima de su capacidad de carga). A su vez, los grandes actores transformadores —en la paradigmática figura de las corporaciones transnacionales-, lejos ya de asumir la situación y revertir la tendencia, mantienen su doctrina de crecimiento mientras proyectan una imagen ambientalmente amigable (eco-friendly). Por último, dicha imagen, materializada mediante piezas publicitarias, puede servir como mecanismo de aprendizaje para formar ciudadanos críticos.

\section{MÉTODo}

En esta contribución se toman como objeto de análisis 17 piezas publicitarias deliberadamente seleccionadas, a partir de las cuales se identifican los mensajes, los contextos en los que estos se producen y las posibles contradicciones en las que puedan incurrir. Se hace uso de una metodología cualitativa basada en la categorización de estas piezas en grandes discursos o enfoques argumentales a partir de un análisis de contenido previo. A su vez, como trasfondo metodológico se recurre también al Análisis Crítico del Discurso (Van Dijk, 2016), por el cual el discurso presente en esta publicidad legitima y reproduce las formas de poder de los lobbies empresariales y las corporaciones transnacionales. Se trata de enfocar el discurso hacia el contexto social, político y, muy particularmente, medioambiental en el que se producen. El enfoque multidisciplinario y flexible de este tipo de análisis discursivo lo hace especialmente propicio como herramienta de partida.

Por un lado, hay que comenzar diferenciando los tipos de mensajes contenidos en un anuncio, discerniendo los significados denotativos u objetivos de los connotativos o implícitos, estos últimos condicionados por el contexto sociocultural del receptor-consumidor. A continuación, el enfoque crítico opera al reconocer la asociación que se establece entre el producto o servicio y la promesa de valoración personal y social vinculada a su consumo. Para ello, se profundiza tanto en la cualidad emotiva de los códigos comunicativos (la estética, el montaje, la música...) como en los atributos persuasivos del mensaje ideológico subyacente (libertad, exclusividad, confort, etc.). El mensaje publicitario, por medio de la persuasión y la interpelación a sentimientos y emociones, dialoga no solo con nuestras necesidades, sino también con nuestro aparato cultural de valores (Arconada, 2007), hasta el punto de que se le otorga mayor protagonismo al valor de marca de un producto que a sus atributos intrínsecos.

Tras estos ejercicios analíticos iniciales, se persigue que el alumnado contraste las ideas y valores proyectados en la publicidad con hechos, cifras y evidencias extraídas de distintas fuentes. Se trata de una tarea exploratoria, de investigación, en la que tienen que consultar referencias tales como las memorias de actividades de las propias compañías, informes oficiales, datos de terceros o noticias en los medios (prensa, televisión, etc.). En esta propuesta se aportan algunas fuentes que pueden servir como pauta orientativa. Por último, la secuencia metodológica concluye con una valoración fundamentada en la que se evalúa el grado de distorsión del mensaje publicitario.

El material de trabajo consta, por su parte, de un conjunto de spots publicitarios televisivos, emitidos entre 2006 y 2017. Se recurre a este tipo particular de soporte audiovisual por varios motivos: por un lado, por la enorme penetración que la televisión aún tiene en la sociedad (87,8 \% de penetración en 2016, según AIMC), a pesar de la intensa competencia que plantea Internet. Este 
fenómeno no pasa desapercibido para las compañías anunciantes, que recurren en primer lugar a la televisión como medio publicitario, también por delante de Internet: la inversión publicitaria en televisión en 2016 ascendió a más de 2100 millones de euros (acaparando un 40,5 \% de todos los medios convencionales), seguida de la inversión en Internet, de 1400 millones de euros (26,9 \%) (Sánchez, 2017) ${ }^{1}$.

Pero también se emplea este soporte puesto que la cultura visual que caracteriza a la sociedad contemporánea — y en especial a la población joven — ha universalizado los lenguajes de los que se vale el anuncio publicitario. Las narrativas audiovisuales que se condensan en ese microrrelato que es un spot de medio minuto se han vuelto algo muy familiar y cotidiano para el público espectador. A ello ha contribuido la estrecha interrelación entre la publicidad y el cine como manifestación paradigmática de cultura popular ${ }^{2}$.

Esta selección no pretende ser una muestra representativa de los discursos publicitarios que de alguna manera aluden al medio ambiente. Se trata de una muestra discrecional (basada en un muestreo no probabilístico), aunque sí muy ilustrativa para poder articular un total de 5 ejes argumentales que sustentan este ejercicio de pensamiento crítico en torno a la retórica medioambiental; más concretamente en el ámbito del sector automovilístico y la movilidad urbana.

Pero ¿por qué prestarle una atención especial al sector del automóvil, en particular cuando se relaciona con el paisaje urbano, el espacio público y la movilidad dentro de la ciudad? Por un lado, por la estrecha relación que guarda con el sector energético, otro de los sectores de gran impacto ambiental, sin el cual los actuales coches de combustión no tendrían razón de ser. A su vez, no hay que menospreciar la contribución del transporte en el calentamiento climático, puesto que en España dicha actividad representa el $24 \%$ de todas las emisiones de GEI, siendo el transporte viario (y dentro de este los turismos) el que más contribuye en la generación de dichas emisiones (Sanz, Vega y Mateos, 2014; Agencia Europea de Medio Ambiente, 2017). Y la relación entre el automóvil y el espacio urbano es tan estrecha que hoy en día el paisaje de las ciudades no se entiende sin la omnipotente presencia del coche. Ya no solo porque estos objetos de consumo transformaron radicalmente los territorios urbanos desde su aparición y popularización a lo largo del siglo XX. También porque el coche representa, en palabras de Riesco y López (2009), “un vector estilístico entre la vida personal y el territorio, una función alentada por la intensa convivencia estética y cultural entre edificios, peatones y coches en la ciudad” (p. 137). Todo ello se refleja en una poderosa maquinaria publicitaria muy al servicio del gremio de los fabricantes de coches. El sector de la automoción es el segundo mayor en volumen de inversión publicitaria en España. Y entre las 20 empresas que más invirtieron en publicidad en los últimos años se encuentran cinco fabricantes: Volkswagen-Audi, Renault, Nissan, Ford y Seat; y dos compañías aseguradoras especializadas: Línea Directa Aseguradora y Mutua Madrileña Automovilística (Sánchez, 2016, 2017).

\section{ANÁLISIS CRÍTICO DEL DISCURSO: LOS EJES ARGUMENTALES}

Los cinco ejes argumentales que sirven como esquema articulador del análisis crítico quedan recogidos en el cuadro I. Tan solo el primero de ellos, que funciona como introducción, incorpora anuncios de otros sectores económicos como el energético, la alimentación o las finanzas. A continuación, se desarrolla con detalle el contenido de cada uno de estos ejes, sirviéndose de mensajes e ideas concretas que se transmiten en las piezas publicitarias que forman la muestra.

\footnotetext{
${ }^{1}$ Hay que tener en cuenta que en las cadenas de las dos principales plataformas televisivas privadas, Mediaset España y Atresmedia, en 2016 los spots publicitarios han ocupado entre un 12,5\% y un $13 \%$ de la franja televisiva, lo que equivale a unos 7,5-7,8 minutos de publicidad por hora de emisión (Optimedia, 2016).

${ }^{2}$ Como pone de manifiesto el que en muchas ocasiones reconocidos directores de cine hayan prestado sus servicios para rodar anuncios publicitarios.
} 
TABLA 1. Ejes argumentales y sus correspondientes anuncios

\begin{tabular}{|c|c|c|c|c|}
\hline Eje Argumental & $\mathrm{N}^{\mathrm{o}}$ & Anuncio & Duración & $\begin{array}{l}\text { Enlace al } \\
\text { Recurso }\end{array}$ \\
\hline \multirow{3}{*}{$\begin{array}{l}\text { 1. La banalización } \\
\text { del término } \\
\text { sostenible }\end{array}$} & 1 & $\begin{array}{l}\text { Knorr, Crema de Verduras } \\
\text { Mediterráneas (2014) }\end{array}$ & $20 \mathrm{~s}$ & $\frac{\text { https://goo.gl/ }}{2 \text { hwT7k }}$ \\
\hline & 2 & $\begin{array}{l}\text { Iberdrola: Hablemos del tiempo } \\
\text { (2017) }\end{array}$ & $46 \mathrm{~s}$ & $\frac{\text { https://goo.gl/ }}{\text { DrvEp3 }}$ \\
\hline & 3 & $\begin{array}{c}\text { Bankia, Préstamo Sostenible: Gotelé- } \\
\text { El edén perdido; Grapadora-La } \\
\text { bestia impasible (2017) }\end{array}$ & $41 \mathrm{~s} ; 43 \mathrm{~s}$ & $\begin{array}{l}\frac{\text { https://goo.gl/ }}{\underline{\text { mpe153 }}} \\
\frac{\text { https://goo.gl/ }}{\text { C8aroa }}\end{array}$ \\
\hline \multirow{4}{*}{$\begin{array}{l}\text { 2. El coche como } \\
\text { "la solución } \\
\text { ambiental" }\end{array}$} & 4 & $\begin{array}{l}\text { Renault Eco2: Hollywood (Ecología } \\
\text { al alcance de todos) (2007) }\end{array}$ & $20 \mathrm{~s}$ & $\frac{\underline{\text { https://goo.gl/ }}}{\underline{\text { m4B2WM }}}$ \\
\hline & 5 & Smart Eco: Vaca (2008) & $20 \mathrm{~s}$ & $\frac{\text { https://goo.gl/ }}{\text { FTDYoJ }}$ \\
\hline & 6 & $\begin{array}{l}\text { Nissan Leaf: ¿Por qué este oso polar } \\
\text { hace este largo viaje? (2010) }\end{array}$ & $1 \mathrm{~min}$ & $\frac{\underline{\text { https://goo.gl/ }}}{\text { dbZuke }}$ \\
\hline & 7 & $\begin{array}{l}\text { Renault Z.E. eléctrico: Vida eléctrica } \\
\text { (2011) }\end{array}$ & $1 \mathrm{~min} 4 \mathrm{~s}$ & $\frac{\text { https://goo.gl/ }}{\text { ZAfNjy }}$ \\
\hline \multirow{3}{*}{$\begin{array}{l}\text { 3. El menosprecio } \\
\text { de otros medios } \\
\text { de transporte }\end{array}$} & 8 & $\begin{array}{l}\text { Seat Altea: Respeta tu espacio vital } \\
\text { (2006) }\end{array}$ & $30 \mathrm{~s}$ & $\frac{\text { https://goo.gl/ }}{\text { hC7AQ9 }}$ \\
\hline & 9 & $\begin{array}{l}\text { Línea Directa Aseguradora, seguro de } \\
\text { ciclomotores (2012) }\end{array}$ & $30 \mathrm{~s}$ & $\frac{\text { https://goo.gl/ }}{\text { JsBS39 }}$ \\
\hline & 10 & $\begin{array}{l}\text { BMW: Cuando conduzcas, conduce } \\
\text { (2016) }\end{array}$ & $45 \mathrm{~s}$ & $\frac{\underline{\text { https://goo.gl/ }}}{\text { KNJ4Jk }}$ \\
\hline \multirow{4}{*}{$\begin{array}{c}\text { 4. La } \\
\text { trivialización de } \\
\text { la ciudad y la } \\
\text { complejidad } \\
\text { urbana }\end{array}$} & 11 & $\begin{array}{c}\text { Nissan Qashqai: Urbanproof (spot } \\
\text { Skate); (spot Play with the city) } \\
\text { (2010) }\end{array}$ & $41 \mathrm{~s} ; 31 \mathrm{~s}$ & $\begin{array}{l}\frac{\text { https://goo.gl/ }}{2 \text { XqTRU }} \\
\frac{\text { https://goo.gl/ }}{\underline{\text { sLkBN8 }}}\end{array}$ \\
\hline & 12 & $\begin{array}{c}\text { Nissan gama crossover: Escribe } \\
\text { ahora tu historia (2015) }\end{array}$ & $40 \mathrm{~s}$ & $\frac{\underline{\text { https://goo.gl/ }}}{\underline{\text { Rkhavs }}}$ \\
\hline & 13 & $\begin{array}{l}\text { Peugeot 2008: La ciudad como nunca } \\
\text { la habías visto (2013) }\end{array}$ & $32 \mathrm{~s}$ & $\underline{\underline{\text { https://goo.gl/ }}} \frac{\underline{66 \mathrm{fDKw}}}{\underline{6}}$ \\
\hline & 14 & $\begin{array}{c}\text { Toyota Auris Hybrid: Reenamórate } \\
\text { de la conducción (2014) }\end{array}$ & $44 \mathrm{~s}$ & $\frac{\text { https://goo.gl/ }}{\text { BZQd1w }}$ \\
\hline \multirow{3}{*}{$\begin{array}{l}\text { 5. La cosmética } \\
\text { tecnológico- } \\
\text { digital }\end{array}$} & 15 & Ford Kuga (2013) & $20 \mathrm{~s}$ & $\frac{\underline{\text { https://goo.gl/ }}}{\underline{\text { DLuFLS }}}$ \\
\hline & 16 & Citroën C3: Marry me (2016) & $31 \mathrm{~s}$ & $\frac{\text { https://goo.gl/ }}{\text { WDRPgN }}$ \\
\hline & 17 & Opel Corsa (2016) & $30 \mathrm{~s}$ & $\frac{\text { https://goo.gl/ }}{\text { aBHrkr }}$ \\
\hline
\end{tabular}

Fuente: elaboración propia 


\subsection{La banalización del término sostenible}

Este primer eje argumental plantea la utilización arbitraria del término sostenible como un concepto vacuo. Se aprovecha su falta de legitimidad científico-técnica, en tanto que no constituye ni una magnitud física ni una variable cuantitativa mesurable, como pudiera ser la temperatura o la tasa de desempleo. Así, se aplica el término como un comodín, hasta banalizarlo y vaciarlo de cualquier contenido verdaderamente ambiental. El anuncio $n^{0} 1$ (Knorr, que también ilustra la introducción de este artículo), hace una alusión clara a "las verduras cultivadas de forma sostenible". Igualmente, el anuncio $\mathrm{n}^{\circ} 2$ (Iberdrola) sitúa la compañía como "líder mundial en sostenibilidad”. En ambos casos, se utiliza esta expresión sin que se realmente se pueda evaluar o cuantificar dicha condición.

El ejercicio de contraste crítico de la información pasa por consultar la documentación sobre el modelo de producción de estas empresas. El Informe Integrado de Iberdrola (2017) muestra que tan solo el 39 \% de toda la producción neta de la compañía procede de fuentes renovables ${ }^{3}$, frente a un $61 \%$ que procede de distintas fuentes no renovables destacando la generación de ciclo combinado (35\%) o la energía nuclear (18\%). Por su parte, el análisis de la campaña de sostenibilidad de Knorr, en particular, y de Unilever, en general, muestra todo un catálogo de buenas intenciones en relación a prácticas agrícolas respetuosas con el medio ambiente, hasta el punto de etiquetar sus productos con el llamativo sello del Sustainability Partnership Knorr. Sin embargo, nada se menciona respecto de alimentos procedentes de la agricultura ecológica, la certificación normativa que oficialmente regula la producción agraria en esos términos.

Por su parte, la pareja de anuncios de Bankia que oferta los Préstamos Sostenibles (anuncio ${ }^{\circ}$ 3) muestra, en un ingenioso ejercicio creativo, un dramático escenario futuro "si no protegemos la naturaleza y el medio ambiente". Que una oferta financiera pensada para la "compra de productos que defienden los valores medioambientales" proceda, precisamente, de esta entidad bancaria, favorece mucho el análisis crítico y el contraste de información. Tan sólo hay que traer a colación los distintos episodios polémicos y mediáticos en los que ha estado involucrada esta entidad y sus distintos responsables: caso Bankia (investigación judicial por la salida a Bolsa de la entidad en 2011), preferentes, tarjetas opacas, reducción drástica de plantilla, etc.

\subsection{El coche como "la solución ambiental"}

Este segundo eje argumental reúne distintos anuncios con un discurso común: lejos de representar uno de los mayores responsables del actual deterioro ambiental, el coche es aquí presentado como una solución ambiental per se. El anuncio $\mathrm{n}^{\circ} 4$ constituye la quintaesencia del giro publicitario que en torno a 2007-2008 experimenta la publicidad del sector del automóvil, cuando surgen multitud de campañas que proyectan la idea de coches que son respetuosos con el medio ambiente. En este caso, como en algunos otros someramente tratados en Camps Monsech (2010), se presenta el Renault Megane Eco2 bajo la llamativa denominación de coche ecológico (junto al eslogan la ecología al alcance de todos). Esta afirmación tan rotunda —podría considerarse un oxímoron- se justifica por su reducción de emisiones de GEI, lo que, en cualquier caso, no convierte a este vehículo en ecológico, sino tan solo en menos contaminante. Similar argumento utiliza la campaña publicitaria de Smart Eco (anuncio $n^{\circ}$ 5). Amparándose en un menor consumo de combustible, se sostiene que este coche altera muy poco el medio ambiente. Las referencias a la vaca, pastando en medio del campo, ajena al vehículo que se adivina por detrás, refuerzan este mensaje de respeto por el entorno natural. Estos y otros discursos tienden a situar el coche y la movilidad motorizada como beneficios ambientales tan solo por el hecho de que se han practicado ciertas innovaciones que reducen en alguna medida el impacto que se genera (menos emisiones de

\footnotetext{
${ }^{3}$ Energía renovable no significa sostenible, dado que también se incluye en esta categoría la generación hidroeléctrica, que, pese a ser relativamente limpia, también implica un impacto considerable de los ecosistemas fluviales.
} 
GEI y menos consumo de combustible). Pero contaminar menos no significa ser beneficioso para el medio ambiente. En estos casos, se juega con las paradojas y las ambigüedades que sitúan algo menos malo como algo ya de por sí bueno.

Los anuncios $n^{\circ} 6$ y 7 recurren a este tipo de paradojas por medio del coche eléctrico. En el primero de ellos un oso polar (un animal fetiche por su asociación simbólica con el Ártico, el riesgo del deshielo y el calentamiento global) abraza a un usuario del Nissan Leaf, $100 \%$ eléctrico. El segundo spot muestra alegóricamente cómo resultarían los aparatos eléctricos más cotidianos si, en vez de electricidad, funcionaran mediante un motor de combustión, como sucede con la mayoría de vehículos convencionales. El mensaje figurado resulta claro: el coche de gasolina repercute negativamente en el medio ambiente, pero el coche eléctrico no. La energía eléctrica representa aquí el paradigma de beneficio ambiental, una virtud ecológica que se opone dialécticamente al motor de combustión y los hidrocarburos, única fuente de los males ambientales asociados al coche. Por una razón sólo sencilla en apariencia, que es que el coche eléctrico no emite gases contaminantes.

El ejercicio de análisis crítico de este segundo eje argumental pasa por comprender las fuentes de energía que se utilizan en la generación de electricidad. El balance energético anual en España (Red Eléctrica Española, 2017) muestra que un 60 \% aproximado de la energía eléctrica sigue procediendo de fuentes no renovables (principalmente nuclear y carbón). Es decir, los coches eléctricos sí contaminan, por cuanto que la mayor parte de la energía con la que se mueven procede de fuentes contaminantes — en contra de esa idea falsa pero que está muy extendida—. Con todo, sí hay que reconocer las menores repercusiones negativas, en términos relativos, tanto de los nuevos motores de combustión como del vehículo eléctrico.

\subsection{El menosprecio de otros medios de transporte}

Los anuncios agrupados bajo este epígrafe muestran un mismo mensaje desde distintos enfoques. Se trata de anteponer el uso del coche (o moto) a otras formas de movilidad supuestamente más sostenibles, caso del transporte público o la bicicleta.

Bajo el lema Respeta tu espacio vital (anuncio ${ }^{\circ}$ 8), el spot del Seat Altea muestra algunas escenas de la vida en sociedad en las que distintas personas ven comprometido su espacio vital, cuando esa metafórica burbuja de aire que nos rodea entra en contacto (y conflicto) con burbujas ajenas. Esto sucede muy especialmente en el metro, medio de trasporte popular y lugar social de encuentro entre grupos de distinta condición. Sin embargo, el coche, gracias a su amplio habitáculo, ofrece un espacio de protección y de confort donde salvaguardar nuestro propio espacio vital. De esta representación despectiva del metro se sirve igualmente el anuncio $n^{\circ} 9$, que enumera las características más peyorativas de este transporte (incomodidad, falta de privacidad, malos olores, etc.) para favorecer así el uso de la motocicleta.

La mirada sesgada que el sector de la automoción ha depositado tradicionalmente en el trasporte público —un competidor nato especialmente en los desplazamientos intraurbanos - ha servido para minusvalorar las ventajas de esta forma de movilidad. El confort, la aparente sensación de autonomía e independencia (resumida en la máxima yo conduzco, yo decido), el prestigio social frente a un medio propio de las clases trabajadoras... son argumentos frecuentemente utilizados para privilegiar el transporte privado motorizado frente al público o a los medios no motorizados.

El anuncio $\mathrm{n}^{\circ} 10$, por su parte, representa un ejercicio creativo mucho más sofisticado, en sintonía con la línea publicitaria que ha caracterizado a la marca bávara en los últimos 15 años. En este caso, es la bicicleta la que se erige como solución de movilidad para cubrir el desplazamiento diario entre la casa y el trabajo. La bicicleta representa aquí un medio moderno, eficaz y, en consecuencia, con el que se puede empatizar. Sin embargo, es el coche el que sigue ostentando la capacidad de trasmitir las emociones que solo la conducción motorizada posee. La bicicleta es (tan 
solo) un medio de transporte, pero no trasmite sentimientos. El coche apela a esta cualidad sensible y emocional, para lo cual resulta evocador el eslogan del anuncio: Cuando conduzcas, conduce.

Para analizar con perspectiva crítica este tipo de mensajes se pueden consultar las distintas encuestas de movilidad que elaboran los organismos públicos o apelar a la experiencia propia de los alumnos y alumnas en su desplazamiento cotidiano al centro docente: ¿Cuánto tiempo empleas en llegar a clase?, ¿Cuánto dinero te supone? ¿Qué ventajas e inconvenientes aprecias en cada uno de los medios...? Preguntas de este tipo pueden servir para alimentar un debate sobre la conveniencia del coche frente a otros medios de trasporte. Para muestra, un botón: recientes investigaciones asocian el caminar o el uso de la bicicleta en los desplazamientos cotidianos con una reducción de las enfermedades cardiovasculares y del riesgo de padecer cáncer (Celis-Morales et ál., 2017).

\subsection{La trivialización de la ciudad y la complejidad urbana}

Este eje argumental se centra en aquellos discursos que ponen en relación el coche y la ciudad. Se trata de una vinculación muy estrecha, prácticamente indisociable, por cuanto que el coche es, ante todo, una herramienta al servicio de la vida urbana. Sin embargo, determinados mensajes publicitarios procuran sesgar esta imagen del espacio urbano, convirtiéndolo en un mero escenario marginal a disposición del coche y de la conducción. Por medio de una simplificación semántica, se reduce toda la complejidad de las funciones urbanas para proyectar en su lugar un paisaje bondadoso, antiséptico o incluso naíf.

Esto es bien perceptible en las sucesivas campañas con las que Nissan ha promocionado su gama de vehículos crossover, bajo el lema Urbanproof (un juego de palabras para referirse a algo diseñado "a prueba de la ciudad"). Para esta investigación solo se ha hecho uso de dos spots muy ilustrativos (anuncio $\mathrm{n}^{0}$ 11): en el primero de ellos el coche sirve como monopatín a un gigante skater que hace de la ciudad su terreno de patinaje. Este leitmotiv fantástico se prolonga en el segundo anuncio, en el que la ciudad cobra animación por medio de distintos elementos morfológicos en calles y edificios (andamios, balcones, fachadas, grúas...) para así juguetear con el coche, que se convierte en su objeto-juguete. Los eslóganes presentes en estos y otros anuncios de la campaña inciden en esta idea: La ciudad, su terreno de juego; Diseñado para jugar con la ciudad; Sincronizado con la ciudad... Esta construcción trivializada de la ciudad minimiza el eterno conflicto que supone la impronta del coche y de la movilidad motorizada: problemas de congestión, contaminación, reducción del espacio público y peatonal en detrimento del espacio rodado, etc.

En una línea similar se sitúa un spot de Nissan (anuncio $\mathrm{n}^{\circ} 12$ ) en el que se representan territorios naturales y urbanos como las páginas de un libro que el conductor va pasando. Bajo el eslogan Escribe ahora tu historia, el contenido connotativo de este mensaje hace referencia a la capacidad del conductor de modificar el medio natural y el medio urbano en su propio beneficio. También aquí es el territorio con sus distintos accidentes (montañas, calles, túneles) los que deben adaptarse al coche, y no al revés. Los primeros deben estar al servicio, a disposición, de este último. La idea de control y dominación del medio, tan presente en el paradigma tecno-científico desde la primera Revolución Industrial, está presente con fuerza aunque no de forma explícita y directa. Así, conviene discutir el papel antropocéntrico del ser humano - en especial el hombre blanco occidental—, y si es legítima su facultad y capacidad para dominar la naturaleza y configurar el entorno según su propia voluntad (Leiss, 1972; Arcury, Johnson y Scollay, 1986; Picas, 2003; Torres y Lobera, 2017).

El anuncio $\mathrm{n}^{0} 13$ continúa con esta idea. El recurso metafórico de representar un trasfondo urbano sobre la aparente forma del desierto se termina de comprender gracias a eslogan final: $L a$ ciudad como nunca la habías visto ${ }^{4}$. El desierto se utiliza como símbolo de la frontera y límite alegórico entre lo domesticado y lo salvaje. Así, conducir este Peugeot es, incluso por el interior de la ciudad, una experiencia que transciende los convencionales límites de lo cotidiano para

\footnotetext{
${ }^{4}$ El eslogan es aún más contundente en la versión argentina del spot: Que el camino se adapte.
} 
adentrarse de manera estimulante en lo indómito. De nuevo la ciudad sufre una transfiguración que la despoja de cualquier atributo no deseado: vulgar, monótona, aburrida..., una metamorfosis en la que el coche funciona como catalizador.

Por último, el anuncio $\mathrm{n}^{\circ} 14$ constituye otro ejercicio atrevido de creación publicitaria, puesto que rompe con los códigos no escritos de la profesión al utilizar un elemento tabú de la publicidad automovilística como es el atasco. Sin embargo, lejos de representar una imagen real, el anuncio viene a transmitir la idea ilusoria de que incluso en los caóticos centros urbanos de las ciudades del Tercer Mundo se puede gozar de un atasco (mientras se esté conduciendo un Toyota Auris). El mensaje final pretende hacernos ver que la satisfacción es aún mayor en "una carretera un poquito mejor”, en una alusión explícita a las desarrolladas infraestructuras de Occidente. Pese a lo valiente de la propuesta, el análisis crítico de este anuncio pasa necesariamente por cuestionar que un atasco suponga una experiencia placentera en cualquier parte del mundo. Especialmente si entre las ciudades con mayores problemas de congestión se encuentran las grandes urbes del mundo desarrollado: en 2016 cuatro ciudades norteamericanas, junto con Moscú, Londres y París, han ocupado el top-ten de ciudades con mayor número medio de horas perdidas en un atasco (Cookson y Pishue, 2017).

En suma, el debate acerca a alumnos y alumnas a una reflexión sobre los usos y las funciones que hacen del espacio urbano un lugar complejo, poliédrico. Dentro de este, los espacios públicos - esto es, la calle y la plaza - siempre han representado los escenarios centrales de convivencia y de conflicto. Por lo tanto, cualquier intento de edulcorar su imagen supone un empobrecimiento de la riqueza social de la vida urbana, privilegiando el papel del coche por encima de otros elementos urbanos o de sus propios inconvenientes.

\subsection{La cosmética tecnológico-digital}

Este último eje argumental pretende cuestionar ciertos aditamentos tecnológicos que son mostrados como verdaderos avances de calado cuando realmente no suponen una innovación técnica sustancial ni en el campo de la mecánica, ni en el de la seguridad, ni en la reducción de los impactos ambientales, etc.

Puede tratarse de un mecanismo para abrir el maletero sin necesidad de usar las manos, con un simple gesto con el pie (anuncio $\mathrm{n}^{\circ} 15$ ); o bien que el coche tenga integrada una videocámara (anuncio $\mathrm{n}^{\circ} 16$ ); o la disponibilidad de una red wifi con tecnología 4G (anuncio $\mathrm{n}^{\circ} 17$ ). En cualquier caso, quedan muy lejos de ser innovaciones técnico-científicas que revolucionen el mundo de la automoción, como sí han hecho otros avances significativos, caso de los motores más eficientes y menos contaminantes, mecanismos de seguridad como el airbag, etc. Poder abrir en maletero con el pie en lugar de con la mano no mejora radicalmente la vida de las personas. Tener una conexión a Internet en el coche cuando ya de por sí se tiene conexión en el teléfono móvil no mejora la calidad de vida de las personas... Así se podría seguir con multitud de ejemplos, de los que aquí solo se aporta una pequeña muestra.

Una lectura crítica de este tipo de contenidos debe servir para reflexionar sobre el verdadero significado de la Tecnología, como conjunto de saberes y conocimientos pensados para satisfacer las necesidades de la sociedad y mejorar sus niveles de bienestar. Las grandes compañías multinacionales recurren a la estrategia de revestir cualquier producto con una aureola de innovación tecnológica con el simple acto de incorporar algún aparato digital (gadget), lo que aquí se ha denominado cosmética tecnológico-digital. Pero ello no significa que represente una aportación tecnológica. Un artefacto digital no es una innovación técnica por sí misma, si bien esta asociación conceptual resulta muy clara, en apariencia, en estos y otros anuncios similares.

En el mundo del automóvil, los últimos pasos verdaderamente revolucionarios han venido más bien de la mano de nuevas formas de interrelación entre usuarios vinculadas al consumo colaborativo: flotas públicas de alquiler de coches eléctricos (carsharing), alquiler de vehículos 
entre particulares, uso compartido de los desplazamientos, etc. Estas innovaciones de uso, si bien son deudoras de las innovaciones digitales asociadas a Internet, representan una nueva forma de entender la movilidad que no implica la adquisición de un nuevo producto, como quiere transmitir una industria que vive de ello. Más bien al contrario, el avance radica en el maximizar el aprovechamiento de los bienes de consumo, favoreciendo el usufructo de un coche frente a su posesión.

\section{A MODO DE CONCLUSIÓN}

Llegados a este punto, cabe formular algunas reflexiones que, a modo de conclusión, sirvan para recapitular sobre el significado de este análisis crítico del discurso publicitario.

Por un lado, se ha querido poner de manifiesto que esta contribución, en el ámbito de la Educación Ambiental, sirve también como vehículo de aprendizaje y reflexión sobre temas medioambientales capitales, que deberían estar presentes en casi cualquier contexto educativo: cambio climático, modelo energético, movilidad urbana, hábitos saludables y su relación con el sedentarismo... Superando así esos contenidos que (aunque necesarios) resultan ya limitados, caso del reciclaje o el uso de bombillas de bajo consumo. En ese sentido, el protagonismo que aquí ha adquirido el coche permite contemplarlo no solo como un medio de transporte, sino también como un vector condicionante del paisaje urbano y como un objeto de identificación simbólica y apropiación cultural.

Por otra parte, en un escenario aparente de libre competencia comunicativa, se observan disimetrías entre interlocutores cuando unos pocos se "apoderan” de los canales comunicativos para monopolizar su discurso — como se ha tenido ocasión de comprobar-, merced a su fortaleza económica. La actividad publicitaria, cuyo fin último consistente en vender cosas, apela a los sentimientos para instalar en la opinión pública un relato que es más emotivo que objetivo (una posverdad), propio de la posmoderna sociedad actual. Al margen del debate sobre la necesaria regulación de los contenidos publicitarios, desde los ámbitos educativos nos corresponde formar a alumnos y alumnas en una mirada crítica, incluso escéptica, ante estos mensajes. En esta contribución se ha hecho uso de estudios, informes y otras fuentes de información como instrumentos de contraste con los que calibrar dicho relato para hacerlo más objetivo.

Sin embargo, no hay que menospreciar el papel pedagógico de la publicidad. Eso sí, siempre y cuando — recuperando las reivindicaciones de Pedrós y Martínez (2010) y de Calero, Vilches y Gil (2013) — se desarrolle en los discentes una capacidad crítica de análisis que incremente la competencia y alfabetización mediática en general y publicitaria en particular. Esta contribución no pretende censurar la función de la publicidad como mecanismo de estímulo al servicio del modelo económico de mercado. Como ya manifestara Codeluppi (2007), la publicidad no es buena ni mala en sí misma, sino que depende tanto de su regulación disciplinaria como de los actores sociales (familia y escuela) que deben tutelar su adecuada asimilación.

La publicidad, al fin y al cabo, tiene su lugar en la sociedad de consumo, de la que forma parte consustancial y a la que sirve. Es responsabilidad de los docentes formar ciudadanos que piensen de forma crítica, proporcionándoles herramientas para que sepan interpretar la publicidad en un contexto cultural en el que irremediablemente son parte de la sociedad como consumidores. También en un contexto económico que necesita cambiar el actual modelo de producción y consumo si se quiere hablar — solo entonces — en términos verdaderamente sostenibles. 


\section{Referencias}

Agencia Europea De Medio Ambiente [European Environment Agency] (2017). EEA greenhouse gas-data viewer. Recuperado de http://www.eea.europa.eu/data-and-maps/data/dataviewers/greenhouse-gases-viewer [22 de noviembre de 2017].

Aimc (Asociación Para La Investigación De Medios De Comunicación) (2016). Encuesta General de Medios. Resumen General. Febrero a noviembre 2016. Recuperado de http://www.aimc.es/-Datos-EGM-Resumen-General-.html [22 de noviembre de 2017].

Álvarez, A. y Reyes, M. I. (2011). De la publicidad espectáculo a los valores emocionales: el sector de la energía en España. Área Abierta, 28, 1-24.

Andrés, S. y González, R. (2010). Referencias al medio ambiente en los mensajes publicitarios. Investigación de la publicidad en España entre 2006 y 2007. aDResearchESIC, 2, 6-25.

Aragonés, J. I., Raposo, G. y Izurieta, C. (2001). Las dimensiones del desarrollo sostenible en el discurso social. Estudios de Psicología, 22(1), 23-36. DOI: 10.1174/021093901609578

Arconada, M. Á. (2007). La publicidad interpela nuestros valores (II). Padres y Maestros, 308, 2428.

Arcury, T. A., Johnson, T. P. y Scollay, S. J. (1986). Ecological Worldview and Environmental Knowledge: The "New Environmental Paradigm". The Journal of Environmental Education, 17(4), 35-40.

Calero, M., Vilches, A. y Gil, D. (2013). Necesidad de la Transición a la Sostenibilidad: papel de los medios de comunicación en la formación ciudadana. Didáctica de las Ciencias Experimentales y Sociales, 27, 235-254.

Camps, J. R. (2010). La ecología como argumento de venta en los mensajes publicitarios. Icono 14, A4, 27-38 (Actas del I Congreso Publiradio. Barcelona, 13 y 14 de abril de 2010).

Celis-Morales, C. A., Lyall, D. M., Welsh, P., Anderson, J., Steell, L., Guo, Y. et ál. (2017). Association between active commuting and incident cardiovascular disease, cancer, and mortality: prospective cohort study. British Medical Journal, 357. DOI: $10.1136 / \mathrm{bmj} . j 1456$

Clément, V. (2005). La odisea de un concepto nómada: el desarrollo sustentable en la órbita de la geografía. CUHSO Cultura-Hombre-Sociedad, 10(2), 9-21. DOI: 10.7770/cuhso-V10N2$\underline{\operatorname{art} 311}$

Codeluppi, V. (2007). El papel social de la publicidad. Pensar la publicidad, 1(1), 149-155.

Cookson, G. y Pishue, B. (2017). INRIX Global Traffic Scorecard. Recuperado de http://inrix.com/scorecard/ [22 de noviembre de 2017].

Costanza, R. y Patten, B. C. (1995). Defining and predicting sustainability. Ecological economics, 15(3), 193-196. DOI: 10.1016/0921-8009(95)00048-8

Daly, H. E. (1990). Toward some operational principles of sustainable development. Ecological economics, 2(1), 1-6. DOI: 10.1016/0921-8009(90)90010-R

González, N., Gozálvez, V. y Ramírez, A. (2015). La competencia mediática en el profesorado no universitario. Diagnóstico y propuestas formativas. Revista de Educación, 367, 117-146. DOI: 10.4438/1988-592X-RE-2015-367-284

Hallama, M., Montlló, M., Rofas, S. y Ciutat, G. (2011). El fenómeno del Greenwashing y su impacto sobre los consumidores. Aposta Revista de Ciencias Sociales, 50, 1-38.

Hardoon, D. (2017). An Economy for the 99\%. Oxford: Oxfam GB. Recuperado de https://www.oxfam.org/sites/www.oxfam.org/files/file_attachments/bp-economy-for-99percent-160117-en.pdf [22 de noviembre de 2017].

Heras, F. (2012). El uso de argumentos ambientales en publicidad. Definiendo líneas rojas, reconociendo buenas prácticas. Carpeta Informativa CENEAM, noviembre 2015, 5-11.

Iberdrola, S.A. (2017). Informe Integrado, febrero 2017. Recuperado de https://www.iberdrola.com/wcorp/gc/prod/es_ES/inversores/docs/IA_InformeIntegrado17.pdf [22 de noviembre de 2017]. 
IPCC (Grupo Intergubernamental De Expertos Sobre El Cambio Climático) (2014). Cambio climático 2014: Informe de síntesis. Ginebra: Organización Meteorológica Mundial.

Keeley, B. (2015). Income Inequality: The Gap between Rich and Poor. Paris: Organización para la Cooperación y el Desarrollo Económico (OCDE). DOI: 10.1787/9789264246010-en

Klein, N. (2000). No logo: Taking Aim at the Branded Bullies. Toronto: Knopf Canada.

Leiss, W. (1972). The Domination of Nature. Nueva York: George Braziller, Inc.

López, J. A. y Méndez, J. A. (1996). Una crítica del concepto de desarrollo sostenible. Iztapalapa, 40, 123-140.

López, L. y Aguaded, M. L. (2015). La docencia sobre alfabetización mediática en las facultades de Educación y Comunicación. Comunicar, 44, 187-195. DOI: $10.3916 / C 44-2015-20$

López, A. T., Andrés, S. y González, R. (2008). Tratamiento del medio ambiente en la publicidad convencional (2006-2007). Valladolid: Universidad de Valladolid; Ministerio de Medio Ambiente y Medio Rural y Marino.

Meadows, D. H., Meadows, D. L., Randers, J. y Behrens, W. W. (1972). The limits to growth. New York: Universe Books.

Millennium Ecosystem Assessment (2005). Ecosystems and Human Well-being: Synthesis. Washington DC: Island Press.

Naciones Unidas (1987). Informe de la Comisión Mundial sobre el Medio Ambiente y el Desarrollo. Nueva York.

Optimedia (2016). Informe Mensual de TV. Septiembre de 2016. Recuperado de http://www.optimedia.es/optimedia-intelligence/informe-mensual-de-television-septiembre2016/ [22 de noviembre de 2017].

Pacheco, M. (2010). El discurso disidente de la contrapublicidad verde. Pensar la Publicidad, 3(1), 55-82.

Pedrós, G. (2005). El Observatorio de la publicidad de la movilidad sostenible y la televisión. Comunicar, 25.

Pedrós, G. y Martínez, P. (2010). Publicidad, educación ambiental y calentamiento global. En F. Heras et ál. (Coords.), Educación ambiental y cambio climático. Respuestas desde la comunicación, educación y participación ambiental (pp. 103-120). Oleiros (A Coruña): Centro de Extensión Universitaria e divulgación ambiental de Galicia.

Picas, J. (2003). Tecnociencia y desarrollo. Crítica antropológica a los procesos de transferencia de tecnología al Tercer Mundo. Revista CIDOB d'afers internacionals, 60, 147-159.

Prody, J. M. (2016). Combating greenwashing through public critique. Communication Teacher, 30(2), 94-99. DOI: 10.1080/17404622.2016.1139151

Red Eléctrica Española (2017). Informe del Sistema Eléctrico Español 2016. Recuperado de http://www.ree.es/sites/default/files/11_PUBLICACIONES/Documentos/InformesSistemaEle ctrico/2016/inf_sis_elec_ree_2016.pdf [22 de noviembre de 2017].

Riesco, P. y López, T. (2009). Automóvil, ciudad y diseño: un fermento estilístico en el paisaje urbano. En C. Tapia y M. Varona (Eds. y coords.), Actas de los seminarios de apoyo a la investigación Hibridación y Transculturalidad en los modos de habitación contemporánea. El territorio andaluz como matriz receptiva (pp. 137-146). Sevilla: Universidad de Sevilla.

Ruiz, C. y Conde, E. (2002). El uso del medio ambiente en la publicidad. Medio ambiente y comportamiento humano, 3(1), 89-101.

Sánchez, M. Á. (2016). Resumen estudio INFOADEX de la inversión publicitaria en España 2016. Recuperado de http://infoadex.factoriadigitalpremium.es/infoadex3/documentacion/ RESUMEN2016.pdf [22 de noviembre de 2017].

Sánchez, M. Á. (2017). Resumen estudio INFOADEX de la inversión publicitaria en España 2017. Recuperado de http://infoadex.factoriadigitalpremium.es/infoadex3/documentacion/ ESTUDIO-COMPLETO-2017.pdf [22 de noviembre de 2017]. 
Sanz, A., Vega, P. y Mateos, M. (2014). Las cuentas ecológicas del transporte en España. Madrid: Libros en Acción ( $2^{\mathrm{a}}$ ed.).

Terrachoice Group Inc. (2009). The seven sins of greenwashing. Environmental Claims in Consumer Markets. Recuperado de http://sinsofgreenwashing.com/index3c24.pdf [22 de noviembre de 2017].

Terrachoice Group Inc. (2010). The Sins of Greenwashing: Home and Family Edition. Recuperado de http://sinsofgreenwashing.com/index35c6.pdf [22 de noviembre de 2017].

Torres, C. y Lobera, J. A. (2017). El declive de la fe en el progreso. Posmaterialismo, ideología y religiosidad en las representaciones sociales de la tecnociencia. Revista Internacional de Sociología, 75(3), e069. DOI: 10.3989/ris.2017.75.3.16.61

Unilever (2016). Unilever Annual Report and Accounts 2016. Recuperado de https://www.unilever.com/Images/unilever-annual-report-and-accounts-2016_tcm244498880_en.pdf [22 de noviembre de 2017].

Van Dijk, T. A. (2016). Análisis Crítico del Discurso [Critical Discourse Analysis]. Revista Austral de Ciencias Sociales, 30, 203-222.

Vos, J. (2009). Actions speak louder than words: Greenwashing in corporate America. Notre Dame Journal of Law, Ethics \& Public Policy, 23(2), 673-697.

WWAP (United Nations World Water Assessment Programme) (2014). The United Nations World Water Development Report 2014: Water and Energy. Paris: UNESCO.

CÓMO CITAR ESTE ARTÍ́CULO

García Martín, M. (2018). ¿Te gusta conducir? Una mirada crítica desde la publicidad de coches y su retórica medioambiental. Didáctica de las ciencias experimentales y sociales, 34, 91-104. DOI: $10.7203 /$ DCES.34.11008. 